5 Dec 2005 Track changes by PB

\title{
Understanding suicidal ideation in psychosis: findings from the prevention of relapse in psychosis trial
}

\author{
Laura Fialko \\ Department of Psychology, Institute of Psychiatry, King's College London, University \\ of London, UK. \\ Daniel Freeman \\ Department of Psychology, Institute of Psychiatry, King's College London, University \\ of London, UK. \\ Philippa A. Garety \\ Department of Psychology, Institute of Psychiatry, King's College London, University \\ of London, UK. \\ Elizabeth Kuipers \\ Department of Psychology, Institute of Psychiatry, King's College London, University \\ of London, UK. \\ Paul E. Bebbington \\ Department of Mental Health Sciences, UCL UK. \\ Graham Dunn \\ Biostatistics Group, School of Epidemiology \& Health Sciences, University of \\ Manchester, UK. \\ David Fowler \\ School of Medicine, Health Policy and Practice, University of East Anglia, UK.
}

Draft $5^{\text {th }}$ December 2005

Text 4098 words 


\section{INTRODUCTION}

The high incidence of suicidal behaviour in schizophrenia is well-documented. The lifetime rate of completed suicide is estimated to be around 10\% (Caldwell and Gottesman, 1990). Estimates of non-fatal suicide attempts range from 18-55\% (Roy et al., 1986; Radomsky et al., 1999). Further, 40-54\% of individuals with schizophrenia either make an attempt or think about ending their lives (McGlashan, 1984; Radomsky et al., 1999).

There is substantial evidence demonstrating a relationship between these different suicidal expressions. Completed suicide in psychosis has been linked to past suicide attempts, and also to past and recent suicidal ideation (Hawton et al., 2005). Among studies that have investigated a range of social, psychological and clinical risk factors for suicide in psychosis, past suicidal behaviour has regularly been identified as one of the most reliable predictors (Nordentoft et al., 2002; De Hert et al., 2001; Walsh et al., 2001). Joiner et al. (2005) suggest that less severe suicidal behaviours may be a necessary precondition for the act of completed suicide, as these enable individuals to become competent at self-injuring, and to habituate to the negative aspects of suicide, such as fear, pain and taboo.

On this basis, suicidal ideation can be regarded as an 'early warning' for more severe suicidal behaviour, making it an important phenomena to identify in vulnerable individuals, as well as a potential target for intervention. Gaining a clear understanding of the causes of suicidal thoughts in individuals with psychosis is an important step towards this therapeutic goal.

Existing studies of suicide in psychosis have focused on socio-demographic and clinical predictors. Male gender, younger age, frequent relapses and recent loss events are well-known to be associated with an increased suicide risk (see Caldwell and 
Gottesman, 1990; Hawton et al., 2005). There has, however, been less attention paid to the cognitive and emotional processes underlying suicidal behaviour in psychosis. Only depression and hopelessness have been reliably identified as risk factors (Drake et al., 1986; Dassori et al., 1990; Addington and Addington, 1992). There is a need for consideration of other affect-related processes, such as self-esteem and schematic beliefs. These have been identified as aetiological and maintaining factors in studies of depression (Beck, 1967; Clark, Beck and Alford, 1999), and may play a similar role in depressed mood and suicidal thinking in psychosis.

Sucidality might equally be driven by the positive symptoms of psychosis. There are several reasons to expect hallucinations and delusions to be specific risk factors (Siris, 2001), including a tendency for irrational thinking and behaviour when positive symptoms are pronounced, and intentional acts of suicide taken for psychotic reasons (for example to escape imagined persecution). Findings concerning the suicide risk associated with hallucinations and delusions have however been varied (Fenton et al., 1997; Grunebaum et al., 2001; Nordentoft et al., 2002; Stephens et al., 1999; Walsh et al., 2001). This may in part be explained by evidence that individual positive symptom types relate differently to suicidal behaviour: thus, paranoid delusions have been shown to be associated with an increased risk of suicide (Fenton, 2000) but somatic delusions with a decreased risk (Nordentoft et al., 2001). Further, the experience of psychotic symptoms can be seen as multidimensional (Garety and Hemsley, 1994). For instance, delusional experience differs in levels of belief conviction, preoccupation and distress. Aspects like delusional distress may relate more closely to suicidal ideation. Existing studies have not looked at suicidal ideation in relation to the multidimensional nature of psychosis. 
The current study investigates individual hallucination and delusion types in relation to suicidal ideas, but also takes a multidimensional approach to psychotic symptoms. This enables symptom severity and subjective distress to be investigated as separate risk factors. The study is a secondary analysis based on data from the Psychological Prevention of Relapse in Psychosis (PRP) trial, a randomised control trial of cognitive behavioural therapy for the positive symptoms of psychosis.

We aimed to identify the prevalence of suicidal thoughts in a group of individuals with relapsing psychosis, to describe demographic and clinical factors associated with suicidal ideation, to investigate the beliefs and emotions associated with suicidal thinking, and to examine the relationship of suicidal behaviour to psychotic experience..

We predicted that suicidal ideation in psychosis would not be associated with psychotic symptoms per se. It would instead be driven by the affective responses to delusions and hallucinations, by depressed mood, and by other key processes related to affect, including low self-esteem, and negative views about self, others and the illness itself.

\section{METHOD}

\section{Participants}

The participants were 290 individuals recruited to the Psychological Prevention of Relapse in Psychosis (PRP) Trial (ISRCTN83557988). The PRP trial is a UK multicentre randomised controlled trial of cognitive behaviour therapy and family intervention for psychosis, designed to investigate both therapeutic outcome and the psychological processes associated with psychosis.

The trial is located in four NHS Trusts in London and East Anglia. Recruitment was from specified clinical teams, both inpatient and outpatient services, with the aim of 
obtaining a representative sample of individuals with psychosis. Patients meeting the eligibility criteria were asked to provide informed consent for participation by a trial research worker or research clinical psychologist. Patients were recruited at the time of a relapse in positive symptoms. For patients with persistent symptoms there had to be a significant exacerbation in positive symptoms (which most often led to hospital admission). The inclusion criteria were: current diagnosis of non-affective psychosis (schizophrenia, schizo-affective psychosis, delusional disorder; ICD-10, F20), age 1865 years, relapse occurring not more than 3 months before consent to enter the trial, rated at least 4 (moderate severity) on the PANSS on at least one positive psychotic symptom at first time of meeting, and completion of the BDI suicide item (item 9, see below). Ninety six percent of the 301 PRP participants completed the BDI suicide item.

\section{Measures}

The baseline assessment was completed by a trial research worker after patient consent had been obtained. The aim was to complete the assessment within a 3-week period.

\section{Basic demographic and clinical data}

Information on age, sex, ethnicity, diagnosis, length of illness, number of admissions, marital status, presence of a carer, and history of violent behaviour was taken from the clinical notes. Employment status was obtained from the Client Service Receipt Interview (Beecham and Knapp, 1992), a health economic survey.

\section{Suicide measures}

Beck Depression Inventory (BDI-II) Item 9: Item 9 of the BDI is a 4-item scale measuring suicidal ideation over the previous two weeks. Ratings are 1 ("I don't have any thoughts of killing myself), 2 ("I have thoughts of killing myself but I would not carry them out", 3 ("I would like to kill myself") and 4 ("I would kill myself if I had the chance"). This single item has previously been shown to correlate with the Beck Scale 
of Suicidal ideation in both in-patient and out-patient psychiatric samples ( $\mathrm{r}$ 's $=.56-.58$; Beck and Steer, 1991). It also has good predictive validity: Brown (2002) reported that patients scoring 2 or higher on item 9 are 6.9 times more likely to commit suicide than those who scored less than 2 on this BDI item. For the present analysis patients scoring 2 were considered to be a mild suicidal ideation group, on the basis that they were not expressing the desire to act on their ideation. Individuals scoring 3 or 4 were combined to make a severe suicidal ideation group.

SCAN item 6.11- suicide or self-harm: This single item assesses ideation and suicide attempts at the height of relapse, which was often retrospective (up to three months before completion of the baseline assessment. It was therefore not used as the main suicide outcome measure. The ratings on the five-point scale are 0 ("Absent"), 1 ("Deliberate intrusive thoughts but no attempt"), 2 ("Injured self or made attempt, no serious result"), 3 (“Injured self or made attempt, serious result”), 4 (“Made an attempt at suicide designed to result in death").

History of suicide attempts: The dichotomous variable "presence or absence of past suicide attempts" was coded from inspection of the clinical notes. All past attempters, irrespective of number of attempts, severity of injury or intent to die were grouped together and compared with individuals who had shown no past suicidal behaviour.

\section{Emotion and belief measures}

Beck Depression Inventory-II (BDI-II; Beck et al., 1996). The BDI-II is a self-report 21item, four point scale (0-3) for the assessment of depression. Depression is assessed over the past fortnight. The total of all items (except the suicide item) was used. Higher scores indicate higher levels of depression. 
Beck Anxiety Inventory (BAI; Beck et al., 1988). The BAI is a self-report, 21 items, four point (0-3) scale for the assessment of anxiety. Anxiety is assessed over the past week. Higher scores indicate higher levels of anxiety.

Rosenberg Self-esteem Scale (Rosenberg, 1965). The RSES is a 10-item, four point scale (1-4) that assesses current levels of global self-esteem. The total score is usually used. A high total score is indicative of low global self-esteem.

The Brief Core Schema Scale (Fowler et al., in press). The BCSS is a 24-item, five point rating scale (0-4) that assesses specific beliefs about the self and others. Four scores are obtained: negative self (6 items), positive self (6 items), negative others (6 items) and positive others (6 items). The BCSS is a quick self-report measure, rather than a lengthy interview. The detailed psychometric properties are documented elsewhere (Fowler et al., in press). The BCSS showed good internal consistency and Cronbach's alpha coefficients were all $>.78$. Principal components analysis revealed a four component solution with the four sub-scale scores accounting for $57 \%$ of the variance.

Revised Illness Perception Questionnaire (Weinman et al., 1996). The IPQ assesses individuals' views of their current problems/illness. It is split into two sections. The first concerns symptom identity and is a 17 item, 4 point scale for self-report of a range of symptoms (never, occasionally, frequently or all the time). The second section

is a 27-item, 5 point scale investigating degree of agreement with a number of statements about illness causes (11 items), time-line ( 3 items), consequences ( 7 items) and cure control (6 items).

\section{Positive symptom measures}

Scale for the Assessment of Positive Symptoms (SAPS; Andreasen, 1984). The SAPS is 35-item, 6 point (0-5) rating instrument for the assessment of the positive symptoms of 
psychosis. Symptoms are rated over the last month. Scores are obtained for hallucinations (7 items), delusions (13 items), bizarre behaviour (5 items), positive formal thought disorder (9 items) and inappropriate affect (single item). Individual hallucination and delusion types were dichotomised, with a rating of 2 (mild) or above needed for the symptom to be considered present.

Positive and Negative Syndrome Scale (PANSS; Kay, 1991). The PANSS is a 30-item, seven point (1-7) rating instrument developed for the assessment of phenomena associated with schizophrenia. Symptoms over the last 72 hours are rated. Four scores are obtained: Total (30 items), Positive Scale (7 items), Negative Scale (7 items), and General Psychopathology (16 items).

Psychotic Symptom Rating Scales (Haddock et al., 1999). The PSYRATS is a 17-item, 5-point scale (0-4) multidimensional measure of delusions and auditory hallucinations. Symptoms over the last week are rated. Dimensions of auditory hallucinations comprised frequency, duration, location, loudness, beliefs about origin, negative content, intensity of negative content, amount of distress, intensity of distress, disruption of life and continuation. Dimensions of delusions comprised amount of preoccupation, duration of preoccupation, conviction, amount of distress, intensity of distress and disruption of life.

\section{Statistical methods}

Analyses were conducted using SPSS for Windows (version 12.0.1) (SPSS, 2003). In order to test associations with suicidal ideation, the three suicidal ideation groups (none, low and severe) were compared on all of the above measures. One-way analysis of variance was used for continuous variables and chi-squared tests for categorical variables. For continuous variables where assumptions for parametric tests were broken, analysis was repeated using Kruskal-Wallis tests; parametric test statistics are reported 
however, as results were unchanged in all cases. For categorical, ordered but noncontinuous data (e.g. SAPS individual items) non-parametric test results are reported. Significance test results are all quoted as two-tailed probabilities.

\section{RESULTS}

\section{Clinical and Demographic data}

The group consisted of 290 people. Of these $71 \%$ were male $(n=206)$ and $29 \%(n=84)$ were female. The mean age of the group was 37.6 years $(\mathrm{SD}=10.9)$, with a range from 18-65 years. The sample was drawn from the following ethnic groups: White $(n=207)$, Black-African $(n=28)$, Black Caribbean $(n=23)$, Black-Other $(n=7)$, Indian $(n=5)$ and Other $(n=20)$. Only ten percent of the sample $(n=30)$ were married or in a stable relationship, the remainder being single $(n=210)$, separated/divorced $(n=44)$ or widowed/unknown $(n=6)$. Employment status of the sample was: Employed full or parttime $(n=27)$, student $(n=10)$, unemployed $(n=232)$, retired $(n=9)$ and other $(n=12)$. The clinical diagnoses of the patients were schizophrenia (F20) $(n=247)$, schizo-affective disorder (F25) (n=39) and delusional disorder (F22) (n=4). The mean length of illness was 10.8 years $(\mathrm{SD}=8.8)$, with a mean of 2.5 hospital admissions in the past five years $(\mathrm{SD}=2.2)$. Sixty seven percent were in-patients at the time of the index relapse.

\section{Prevalence of suicidal ideation and behaviour}

Of the 277 patients for whom data was available about past suicidal behaviour, $39 \%$ $(n=107)$ had a history of suicide or self-harm and $61 \%(n=170)$ did not. This figure is in line with the range of rates of non-fatal suicide attempts reported in previous studies (e.g. Roy et al., 1986). Analysis of the SCAN 2.0 showed that $17 \%$ of the sample had made a suicide or self-harm attempt at the height of the recent that led to entry to the trial. 
Rates of current suicidal ideation, measured by BDI item 9 are shown in Table 1. Overall $41 \%$ of individuals reported some degree of suicidal thinking. Those individuals scoring higher on BDI suicidal ideation were more likely to have made a suicide or self-harm attempt at the height of the relapse that preceded entry into the trial, $\chi^{2}(2)=50.907, p<.001$, or over the length of time they had been known to mental health services, $\chi^{2}(2)=15.849, p<.001$.

\section{Table 1 about here}

\section{Socio-demographic factors and suicidal ideation}

The socio-demographic variables examined were age, sex and ethnicity (see Table 2) None of these factors was shown to be associated with degree of suicidal ideation, although when individuals of all non-white ethnicities were grouped and compared to individuals of white ethnicity, a trend was identified towards more suicidal thinking in the white group.

\section{Clinical characteristics and suicidal ideation}

Suicidal ideation was found to be increased in the group with a history of suicide or self-harm. PANSS general psychopathology score was significantly associated with level of suicidal thinking. Finally, individuals consuming alcohol on a daily basis were more likely to express suicidal ideation than those using it less frequently.

\section{Schematic beliefs, emotions and suicidal ideation}

Individuals with suicidal ideation had more severe depression and anxiety, and significantly lower self-esteem than those without suicidal thoughts. Self-esteem and anxiety did not significantly differ between mild and severe suicidal ideation groups, 
but post-hoc Bonferroni tests showed that depression became more severe as degree of suicidal ideation worsened $(p<.001)$.

Results for the three IPQ subscales showed that the suicidal ideation groups had a more negative perception of the consequences of their illness compared to the group who did not show ideation. The severe ideation group also expected their illness to last for a long time and felt less optimistic about the curability or controllability of their illness. IPQ time-line score correlated significantly with length of illness $(r=.173$, $\mathrm{p}=.005$ ), and all three IPQ subscales correlated significantly with PANSS general psychopathology score $(\mathrm{p}<.001)$.

Three of the four subscales of the BCSS were associated with suicidal thinking: The suicidal ideation groups had significantly more negative views and less positive views of themselves reduced in the ideation groups compared to the no ideation group. The severe ideation group also held a more negative view of other people than the group with no suicidal ideation.

\section{Positive symptoms of schizophrenia and suicidal ideation}

There was no significant difference in PANSS positive symptom total between groups, however differences emerged when symptom types and then individual symptoms were investigated using the SAPS (see Table 3).

The SAPS global hallucinations score was associated with suicidal ideation, but the global delusions score was not. Only auditory hallucinations were related to the level of suicidal thinking, being so whether observer-rated or self-reported. The presence of thought disorder was inversely associated with the level of suicidal ideation.

All delusions types present in at least ten percent of the sample were investigated individually. Of these, only two showed a significant relationship with suicidal thinking. Suicidal ideation was greater in people with delusions of guilt, and 
absent in those with grandiose delusions. There was also a trend toward increased suicidal ideation in individuals experiencing delusions of control.

\section{Table 3 about here}

Among the 156 individuals who reported hearing voices during the week of the assessment, four physical characteristics were investigated (frequency, duration, loudness and location) (Table 4). They were not found to relate to suicidal thinking. In contrast, the amount and degree of negative content of the voices, the amount of time the voices cased distress, and the intensity of this distress were all significantly associated with suicidal thinking.

\section{Table 4 about here}

Similar findings emerged in relation to delusions, whereby the emotional response was once again uniquely associated with suicidal ideation (Table 5). In the 226 individuals with delusions, suicidal ideation was related to the amount and intensity of distress in response to the delusion. but not to the amount and degree of preoccupation. Delusional conviction was lower in the mild suicidal ideation group than the no suicide and severe suicidal ideation groups, indicating some uncertainty in beliefs among this group.

\section{Table 5 about here}




\section{DISCUSSION}

The PRP study was not designed as a primary study of suicide, and has certain limitations as a consequence. However, the detailed assessment of positive symptoms in combination with the evaluation of beliefs and emotions in a large clinical sample provides an opportunity to examine the contribution of these factors to suicidal thinking in psychosis. The single item suicide measure is clearly a limitation, despite its ability to discriminate meaningfully. Moreover, as participants had all consented to a treatment trial, they may not be fully representative of those with psychosis. Finally, the analysis was cross-sectional, inevitably limiting causal inference.

The results confirm that a large proportion of individuals with psychosis experience some degree of suicidal ideation: Thirty two percent reported mild ideation and nine percent reported severe ideation in the two weeks leading up to the interview. This overall rate of $41 \%$ of individuals reporting suicidal ideation is well within the range of other estimates (McGlashan, 1984; Radomsky et al., 1999), and supports the use of BDI items to assess ideation. There were associations of suicidal thoughts at the time of the interview both with suicidal thoughts or self-harm attempts at the height of relapse, and with a history of self-harm generally. It therefore seems that some individuals with psychosis think about suicide persistently.

Several variables distinguished these individuals from those without suicidal thoughts, particularly in relation to emotional characteristics, and differences in beliefs about self and illness. Interestingly, few demographic factors were associated with suicidal ideation, and previously identified risk factors such as younger age, male gender, and frequent relapse were not replicated (Brier and Astrachan, 1984; Caldwell and Gottesman, 1990; De Hert et al., 2001). The nature of our sample may account for these differences: Positive symptom relapse was one of the entry criteria for the trial, 
meaning that there were no first episode cases, and in general the group was characterised by long illness, multiple relapse and multiple in-patient admission. There was a trend towards an association between white ethnic groups and suicidal ideation, which has been previously found (Walsh et al., 2001).

In contrast, suicidal thinking was strongly associated with depression and anxiety, and with negative evaluative beliefs. Thus people with suicidal ideation held more negative views of other people "other people are bad", "other people are harsh", more negative views of themselves "I am bad", "I am unloved", and less positive views of themselves "I am valuable", "I am good". They also held more negative views of their illness, seeing it as more likely to last for a long time, to be less controllable, and to connote more severe negative consequences on their lives than those without suicidal thoughts.

The difference in illness perception could in theory arise from genuine differences in symptom severity between these groups. This was not so for positive symptoms, but negative illness perceptions did correlate with PANSS general psychopathology total. Thus, the group with more negative illness perceptions do not suffer more psychotic symptoms, but may endure a wider range of problems in addition to, or as a consequence of, their psychosis. Higher IPQ time-line score (having a more pessimistic view of how long problems are anticipated to last) correlated with actual length of illness, further indicting that illness perceptions correspond to objective circumstances. The relationship between illness perceptions and suicidal ideation may therefore have a rational element. This corroborates with Williams (1997) "cry of pain" concept of suicidal behaviour, in which suicidal behaviour is thought of as an attempt to escape from feelings of entrapment. 
The nature of the beliefs and emotions we have identified in individuals with suicidal ideation allows us to go beyond of depression as a risk factor for suicide (Drake et al., 1986; Dassori et al., 1990; Addington and Addington, 1992) and to provide a profile of the ideas about self and about illness contributing to this depressed mood and accompanying suicidal thoughts. Our findings carry an important clinical implication: interventions aimed at altering negative schematic beliefs and low self-esteem, and coping with illness and its consequences may be successful in reducing suicidal thoughts. Of further therapeutic relevance is the finding that daily alcohol consumption is associated with suicidal thoughts. This habit should be carefully assessed and targeted in interventions, as people abusing alcohol may also risk acting impulsively on their suicidal ideas (Mann et al., 1999; Hufford, 2001).

Our results support the suggestion that suicidal thinking in psychosis is understandable and affect-driven, rather than being of "psychotic" or inexplicable origin. Indeed, thought disorder was actually identified as a protective factor against suicidal thinking: the idea of "irrational" suicide (Siris, 2001) was therefore not supported in our sample. Neither global hallucination nor global delusion scores were identified as risk factors, but there were some significant relationships of individual positive symptoms with suicidal thinking. Among hallucination types, auditory hallucinations were unique in being associated with suicidal ideation. More detailed analysis using the PSYRATS demonstrated a clear distinction between the physical and emotional characteristics of voices in their associations with suicidal ideation. Thus the frequency and physical form of the voices were not related to suicidal ideation, but negative content and the degree distress in response to voices were both significantly associated with degree of suicidal ideation. Only two types of delusions were related to level of suicidal ideation: delusions of guilt increased suicidal thinking, while grandiose 
delusions reduced it. As with auditory hallucinations, it seems that is not the presence of delusions, but rather their content that is associated with suicidal ideation. This was supported by the dimensional analysis of delusions, which demonstrated that the only characteristics of delusions to significantly relate to all levels of suicidal thinking were the amount and intensity of distress.

There may also be a role for delusional conviction in suicidal ideation. An interesting finding was that conviction in delusional beliefs was lower in the mild suicidal ideation group than the no suicide and severe suicidal ideation groups. A possible interpretation of this result is that there are different pathways to suicidal ideation: one in which individuals are troubled by uncertainly in their beliefs (which seems to be associated with mild suicidal ideation) and a second route, in which it is certainty about the beliefs that leads to suicidal wishes. Clarifying the nature of this relationship between delusional conviction and suicidal thoughts, and considering interactions with the content of the delusions and delusional distress would be a valuable topic for future investigation.

Overall, our sample of people with positive symptoms of psychosis, the primary risk factor for suicidal thinking appears to be emotional dysfunction. Desire for suicide seems to relate understandably to the low mood, damaged self-esteem, disappointment and distress that are frequent consequences of living with psychosis and the difficulties that accompany it. These emotional difficulties must be detected and addressed if suicidal thoughts and actions are to be reduced. 


\section{ACKNOWLEDGEMENT}

This work was supported by a programme grant from the Wellcome Trust (No. 062452). We wish to thank the patients taking part in the trial and the participating clinical teams in the four NHS Trusts. The study was supported by Camden and Islington Mental Health and Social Care Trust, North East London Mental Health Trust, Norfolk etc Trust, SCARY. 


\section{REFERENCES}

Addington, D.E. and Addington J.M. (1992). Attempted suicide and depression in schizophrenia. Acta Psychiatrica Scandinavica, 85, 288-291

Andreasen, N.C. (1984). The Scale for the Assessment of Positive Symptoms (SAPS). Iowa City, IA: The University of Iowa.

Beck, A.T. (1967). Depression: Clinical, experimental and theoretical aspects. New York: Hoeber.

Beck, A.T., Steer, R.A., \& Brown, G.K. (1996). BDI-II Manual. San Antonio, TX: The Psychological Corporation.

Beck, A.T., Epstein, N., Brown, G. \& Steer, R. (1988). An inventory for measuring clinical anxiety: psychometric properties. Journal of Consulting and Clinical Psychology, 56, 893-897.

Beck, A.T. and Steer, R.A. (1991). Manual for the Beck Scale for Suicidal ideation. San Antonio, TX : Psychological Corporation.

Beecham, J. and Knapp, M.R.J. (1992). Costing psychiatric interventions. In G. Thornicroft, C. Brewin and J. Wing (Eds.) Measuring mental health needs. London: Gaskell. 
Brier, A. and Astrachan, B.M. (1984). Characterisation of schizophrenic patients who commit suicide. American Journal of Psychiatry, 141, 206-209.

Brown, G. K. (2002). A review of suicide assessment measures for intervention research in adults and older adults. Technical report submitted to NIMH under contract no. 263-MH914950. Bethseda, MD: National Institute of Mental Health, (http://www.nimh.nih.gov/suicideresearch/adultsuicide.pdf)

Caldwell, C.B. and Gottesman I.I., (1990). Schizophrenics kill themselves too: A review of risk factors for suicide. Schizophrenia Bulletin, 16, 571-589

Clark, D.A., Beck, A.T. and Alford, B.A.(Eds.)(1999) Scientific foundations of cognitive theory and therapy of depression. New York: Wiley.

Dassori, A.M., Mezzich, J.E. \& Keshavan, M. (1990). Suicidal indicators in schizophrenia. Acta Psychiatrica Scandinavica, 81, 409-413

De Hert, M., McKenzie, K., \& Peuskens, J. (2001). Risk factors for suicide in young people suffering from schizophrenia: a long-term follow-up study. Schizophrenia Research, 47, 127-134.

Drake, R.E. and Cotton, P.G. (1986). Depression, hopelessness and suicide in chronic schizophrenia. British Journal of Psychiatry, 148, 554-559. 
Fenton, W.S., McGlashan, T.H., Victor, B.J. \& Blyler, C.R. (1997). Symptoms, subtype and suicidality in patients with schizophrenia spectrum disorders. American Journal of Psychiatry, 154, 199-204.

Fenton, W. (2000). Depression, suicide, and suicide prevention in schizophrenia. Suicide and life-threatening behaviour, 30 (1), 34-49.

Fowler, D.G., Freeman, D., Smith, B., Garety, P.A., Kuipers, E.K., Bebbington, P.E. \& Dunn, G. The brief core schema scales: psychometric properties and associations with paranoia, depression, and anxiety in normal and psychosis samples. In press.

Garety PA \& Hemsley DR (1994). Delusions: Investigations into the Psychology of Delusional Reasoning. Hove: Psychology Press Maudsley Monographs.

Grunebaum, M.F., Oquendo, M.A., Harkavy-Friedman, J.M., Ellis, S.P., Li, S., Haas, G.L., Malone, K.M., Mann, J.J. (2001). Delusions and Suicidality. American Journal of Psychiatry, 156, 742-747.

Haddock, G., McCarron, J., Tarrier, N., \& Faragher, E.B. (1999). Scales to measure dimensions of hallucinations and delusions: the psychotic symptom rating scales (PSYRATS). Psychological Medicine, 29, 879-889.

Hawton, K., Sutton, L., Haw, C, Sinclair, J., \& Deeks, J. (2005). Schizophrenia and suicide: systematic review of risk factors. British Journal of Psychiatry, 187, 9-20. 
Hufford, M.R. (2001). Alcohol and suicidal behaviour. Clinical psychology review, 21, 797-811.

Joiner, T.E. Jr., et al. (2005). Four studies on how past and current suicidality relate even when "everything but the kitchen sink" is covaried. Journal of Abnormal Psychology, 114 (2), 291-303.

Kay, S. R. (1991). Positive and negative syndromes in schizophrenia: Assessment and research. New York: Brunner/Mazel.

Mann, J.J., Waternaux, C., Haas, G.L. \& Malone, K. (1999). Toward a clinical model of suicidal behaviour in psychiatric patients. American Journal of Psychiatry, 156, 181189.

McGlashan, T. (1984). The Chestnut Lodge follow-up study. Part II: Long-term outcome of schizophrenia and the affective disorders. Arch. Gen. Psychiatry, 41, 586601.

Nordentoft, M. et al. (2002). OPUS study: Suicidal behaviour, suicidal ideation and hopelessness among patients with first episode psychosis. One-year follow-up of a randomised controlled trial. British Journal of Psychiatry, 181 (suppl.43), s98-s106.

Radomsky, E.D., Haas, G.L., Mann, J.J., \& Sweeney, J.A., (1999). Suicidal behaviour in patients with schizophrenia and other psychotic disorders. American Journal of Psychiatry, 256, 1590-1595. 
Rosenberg, M. (1965). Society and the Adolescent Self-Image. Princeton: Princeton University Press.

Roy, A, (1986). Depression, attempted suicide, and suicide in patients with chronic schizophrenia. The Psychiatric clinics of North America, 9 (1), 193-206.

Stephens, J.H., Pascal, R., \& McHugh, P.R. (1999). Suicide in patients hospitalised for schizophrenia: 1913-1940. Journal of Nervous and Mental Disease, 187, 10-14.

Walsh, E., Harvey, K., White, I., Higgit, A., Fraser, J, \& Murray, R. ( 2001). Suicidal behaviour in psychosis: prevalence and predictors from a randomised controlled trial of case management. British Journal of Psychiatry, 178, 255-260.

Weinman, J., Petrie, K.J., Moss-Morris, R. \& Horne, R. (1996). The illness perception questionnaire: a new method for assessing the cognitive representation of illness. Psychology and Health, 11, 431-445.

Williams, J.M.G. (1997) Cry of Pain: Understanding Suicide and Self-Harm. Harmondsworth: Penguin. 
Table 1: Frequency of suicidal ideation

\section{BDI item 9}

No suicidal ideation:

"I don't have any thoughts of killing myself"

Mild suicidal ideation:

"I have thoughts of killing myself but I would not carry them out"

Severe suicidal ideation:

"I would like to kill myself" or

26

9.0

"I would kill myself if I had the chance" 
Table 2: Characteristics and scores of individuals with no, mild and severe suicidal ideation

\begin{tabular}{|c|c|c|c|c|c|c|c|c|}
\hline Predictor & \multicolumn{2}{|c|}{$\begin{array}{l}\text { No } \\
\text { suicidal } \\
\text { ideation }\end{array}$} & \multicolumn{2}{|c|}{$\begin{array}{l}\text { Mild } \\
\text { suicidal } \\
\text { ideation }\end{array}$} & \multicolumn{2}{|c|}{$\begin{array}{l}\text { Severe } \\
\text { suicidal } \\
\text { ideation }\end{array}$} & \multirow[t]{2}{*}{$\begin{array}{l}\text { F or } \chi^{2} \\
(\mathrm{df}=2)\end{array}$} & $\mathrm{p}$ \\
\hline \multicolumn{8}{|l|}{ Socio-demographic } & \\
\hline & Mean & $\mathrm{SD}$ & Mean & SD & Mean & SD & & \\
\hline \multirow[t]{2}{*}{ Age } & 37.31 & 10.86 & 37.83 & 11.41 & 39.12 & 10.01 & .327 & .721 \\
\hline & $\mathrm{N}$ & $\%$ & $\mathrm{~N}$ & $\%$ & $\mathrm{~N}$ & $\%$ & & \\
\hline Sex (male) & 125 & 60.7 & 62 & 30.1 & 19 & 9.2 & .871 & .647 \\
\hline Sex (female) & 47 & 56.0 & 30 & 35.7 & 7 & 8.3 & & \\
\hline Ethnicity (white) & 115 & 55.6 & 70 & 33.8 & 22 & 10.6 & 4.947 & .084 \\
\hline Ethnicity (non-white) & 57 & 68.7 & 22 & 26.5 & 4 & 4.8 & & \\
\hline \multicolumn{9}{|l|}{ Clinical } \\
\hline & Mean & $\mathrm{SD}$ & Mean & $\mathrm{SD}$ & Mean & SD & & \\
\hline Length of illness & 10.87 & 8.79 & 9.97 & 9.03 & 13.12 & 8.45 & 1.262 & .285 \\
\hline No. admissions in the past $5 \mathrm{yrs}$ & 2.47 & 2.17 & 2.41 & 2.25 & 2.78 & 2.41 & .247 & .781 \\
\hline \multirow[t]{2}{*}{ PANSS general total } & 32.6 & 7.6 & 34.0 & 7.3 & 39.8 & 6.8 & 10.590 & $<.001$ \\
\hline & $\mathrm{N}$ & $\%$ & $\mathrm{~N}$ & $\%$ & $\mathrm{~N}$ & $\%$ & & \\
\hline History of suicide attempt & 51 & 47.7 & 39 & 36.4 & 17 & 15.9 & 15.849 & $<.001$ \\
\hline No history of suicide attempt & 114 & 67.1 & 49 & 28.8 & 7 & 4.1 & & \\
\hline History of violent behaviour & 47 & 57.3 & 26 & 31.7 & 9 & 11.0 & .653 & .721 \\
\hline No history of violent behaviour & 120 & 60.0 & 64 & 32.0 & 16 & 8.0 & & \\
\hline Diagnosis - & 149 & 59.4 & 79 & 31.5 & 23 & 9.2 & .119 & .942 \\
\hline \multicolumn{9}{|l|}{ Schizophrenia/ delusional disorder } \\
\hline Diagnosis - schizoaffective dxer & 23 & 59.0 & 13 & 33.3 & 3 & 7.7 & & \\
\hline Daily alcohol consumption & 25 & 47.2 & 18 & 34.0 & 10 & 18.9 & 9.548 & .008 \\
\hline No daily alcohol consumption & 139 & 61.8 & 72 & 32.0 & 14 & 6.2 & & \\
\hline \multicolumn{9}{|l|}{ Beliefs and emotions } \\
\hline & Mean & $\mathrm{SD}$ & Mean & SD & Mean & SD & & \\
\hline Depression (BDI total) & 16.7 & 11.2 & 26.3 & 10.7 & 37.8 & 9.4 & 55.075 & $<.001$ \\
\hline Anxiety (BAI total) & 16.4 & 13.0 & 26.5 & 14.9 & 25.7 & 14.2 & 17.08 & $<.001$ \\
\hline Self-esteem (RSES total) & 21.1 & 5.7 & 26.2 & 5.9 & 29.4 & 5.8 & 34.163 & $<.001$ \\
\hline IPQ cure-control & 21.6 & 4.2 & 20.5 & 4.3 & 17.4 & 4.6 & 9.904 & $<.001$ \\
\hline IPQ time-line & 9.3 & 3.5 & 10.1 & 3.1 & 11.8 & 2.5 & 6.797 & $=.001$ \\
\hline IPQ consequences & 24.9 & 4.7 & 27.2 & 4.0 & 27.9 & 4.0 & 10.036 & $<.001$ \\
\hline Schema - negative self & 5.2 & 5.2 & 9.2 & 5.7 & 11.9 & 5.6 & 25.489 & $<.001$ \\
\hline Schema - positive self & 11.7 & 6.3 & 8.3 & 6.3 & 6.9 & 5.6 & 10.876 & $<.001$ \\
\hline Schema - negative other & 7.9 & 6.7 & 9.9 & 6.4 & 12.0 & 8.0 & 4.956 & .008 \\
\hline Schema - positive other & 11.1 & 6.1 & 9.6 & 5.8 & 9.4 & 7.0 & 1.900 & .152 \\
\hline
\end{tabular}


Table 3: Positive symptoms and suicidal ideation

\begin{tabular}{|c|c|c|c|c|c|c|c|c|}
\hline Positive symptoms & $\begin{array}{l}\text { No } \\
\text { suicidal } \\
\text { ideation }\end{array}$ & & $\begin{array}{l}\text { Mild } \\
\text { suicidal } \\
\text { ideation }\end{array}$ & & $\begin{array}{l}\text { Severe } \\
\text { suicida } \\
\text { ideatior }\end{array}$ & & $\begin{array}{l}\text { F or } \chi^{2} \\
(d f=2)\end{array}$ & $\mathbf{p}$ \\
\hline \multicolumn{9}{|l|}{ Global scores } \\
\hline & Mean & SD & Mean & SD & Mean & $\mathrm{SD}$ & & \\
\hline PANSS: positive total & 18.1 & 5.8 & 18.0 & 4.7 & 18.2 & 4.3 & .032 & .969 \\
\hline SAPS: global hallucinations & 2.4 & 1.86 & 2.7 & 1.9 & 3.7 & 1.7 & 14.245 & .001 \\
\hline SAPS: global delusions & 3.2 & 1.4 & 3.3 & 1.4 & 3.1 & 1.7 & 1.135 & .567 \\
\hline SAPS: thought disorder & 1.09 & 1.36 & .59 & 1.11 & .35 & .75 & 14.162 & .001 \\
\hline \multicolumn{9}{|l|}{ Delusion type } \\
\hline & $\mathrm{N}$ & $\%$ & $\mathrm{~N}$ & $\%$ & $\mathrm{~N}$ & $\%$ & & \\
\hline Persecutory delusions & 103 & 56.6 & 63 & 34.6 & 16 & 8.8 & 1.913 & .384 \\
\hline No persecutory delusions & 69 & 63.9 & 29 & 26.9 & 10 & 9.3 & & \\
\hline Delusions of guilt & 12 & 30.8 & 21 & 53.8 & 6 & 15.4 & 15.211 & $<.001$ \\
\hline No delusions of guilt & 160 & 63.7 & 71 & 28.3 & 20 & 8.0 & & \\
\hline Grandiose delusions & 66 & 72.5 & 23 & 25.3 & 2 & 2.2 & 12.420 & .002 \\
\hline No grandiose delusions & 106 & 53.3 & 69 & 34.7 & 24 & 12.1 & & \\
\hline Religious delusions & 34 & 57.6 & 22 & 37.3 & 3 & 5.1 & 2.002 & .367 \\
\hline No religious delusions & 138 & 59.7 & 70 & 30.3 & 23 & 10.0 & & \\
\hline Somatic delusions & 35 & 64.8 & 16 & 29.6 & 2 & 5.6 & 1.291 & .524 \\
\hline No somatic delusions & 137 & 58.1 & 76 & 32.2 & 23 & 9.7 & & \\
\hline Delusions of reference & 98 & 55.7 & 62 & 35.2 & 16 & 9.1 & 2.734 & .255 \\
\hline No delusions of reference & 74 & 64.9 & 30 & 26.3 & 10 & 8.8 & & \\
\hline Delusions of control & 22 & 48.9 & 15 & 33.3 & 8 & 17.8 & 5.558 & .062 \\
\hline No delusions of control & 149 & 61.1 & 77 & 31.6 & 18 & 7.4 & & \\
\hline Delusions of mind-reading & 47 & 54.0 & 31 & 35.6 & 9 & 10.3 & 1.373 & .503 \\
\hline No delusions of mind-reading & 124 & 61.4 & 61 & 30.2 & 17 & 8.4 & & \\
\hline Thought broadcast & 23 & 54.8 & 14 & 33.3 & 5 & 11.9 & .658 & .720 \\
\hline No thought broadcast & 148 & 59.9 & 78 & 31.6 & 21 & 8.5 & & \\
\hline Thought insertion & 26 & 51.0 & 20 & 39.2 & 5 & 9.8 & 1.807 & .405 \\
\hline No thought insertion & 145 & 60.9 & 72 & 30.3 & 21 & 8.8 & & \\
\hline \multicolumn{9}{|l|}{ Hallucination type: } \\
\hline Visual hallucinations & 44 & 59.5 & 22 & 29.7 & 8 & 10.8 & .518 & .772 \\
\hline No visual hallucinations & 124 & 58.5 & 70 & 33.0 & 18 & 8.5 & & \\
\hline Auditory hallucinations & 97 & 54.8 & 57 & 32.2 & 23 & 13.0 & 9.606 & .008 \\
\hline No auditory hallucinations & 74 & 66.1 & 35 & 31.3 & 3 & 2.7 & & \\
\hline Olfactory hallucinations & 24 & 49.0 & 18 & 36.7 & 7 & 14.3 & 3.170 & .205 \\
\hline No olfactory hallucinations & 145 & 60.9 & 74 & 31.1 & 19 & 8.0 & & \\
\hline Somatic hallucinations & 43 & 61.4 & 25 & 35.7 & 2 & 2.9 & 4.402 & .111 \\
\hline No somatic hallucinations & 127 & 58.3 & 67 & 30.7 & 24 & 11.0 & & \\
\hline
\end{tabular}


Table 4: Dimensions of auditory hallucinations and their relationship with suicidal thinking

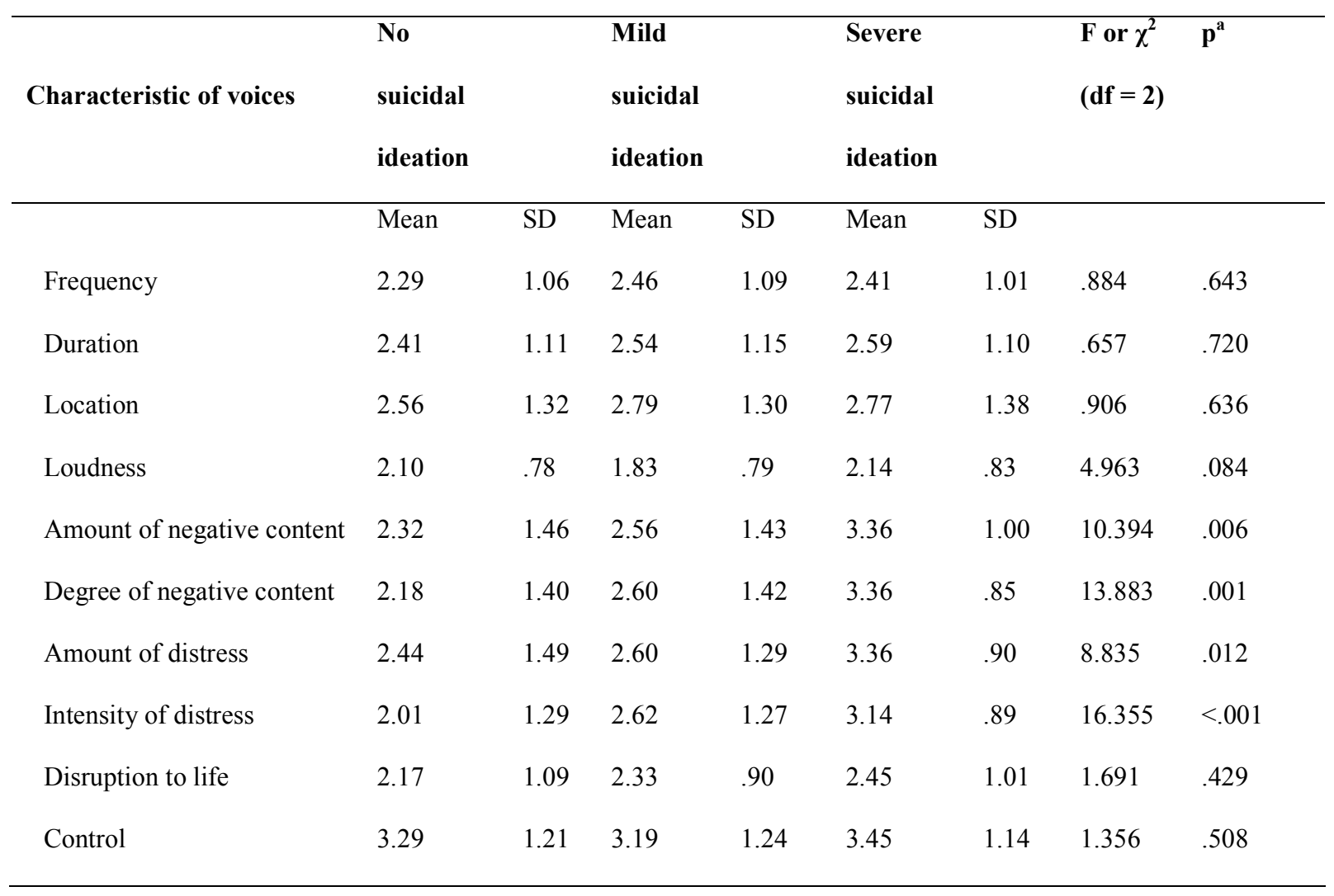


Table 5: Dimensions of delusions and their relationship with suicidal thinking

\begin{tabular}{|c|c|c|c|c|c|c|c|c|}
\hline \multirow{4}{*}{ Characteristic of voices } & \multicolumn{2}{|l|}{ No } & \multicolumn{2}{|l|}{ Mild } & \multicolumn{2}{|l|}{ Severe } & \multirow{3}{*}{$\begin{array}{l}\text { F or } \chi^{2} \\
(d f=2)\end{array}$} & \multirow{3}{*}{$\mathrm{p}$} \\
\hline & \multirow{2}{*}{\multicolumn{2}{|c|}{$\begin{array}{l}\text { suicidal } \\
\text { ideation }\end{array}$}} & \multicolumn{2}{|l|}{ suicidal } & \multicolumn{2}{|l|}{ suicidal } & & \\
\hline & & & ideation & & ideation & & & \\
\hline & Mean & SD & Mean & SD & Mean & SD & & \\
\hline Amount of preoccupation & 2.68 & 1.01 & 2.55 & .99 & 2.71 & 1.19 & .867 & .648 \\
\hline Duration of preoccupation & 2.73 & .96 & 2.74 & .91 & 3.00 & .92 & 1.505 & .471 \\
\hline Conviction & 3.44 & .64 & 3.17 & .78 & 3.52 & .51 & 7.326 & .026 \\
\hline Amount of distress & 2.63 & 1.36 & 2.91 & 1.15 & 3.50 & .83 & 9.296 & .010 \\
\hline Intensity of distress & 2.30 & 1.21 & 2.61 & 1.11 & 2.95 & .80 & 6.481 & .039 \\
\hline Disruption to life & 2.27 & 1.04 & 2.45 & .91 & 2.43 & .93 & 1.201 & .548 \\
\hline
\end{tabular}

